\title{
Metodología para evaluar la madurez de la gestión del conocimiento en algunas grandes empresas colombianas
}

\section{Methodology for assessing the maturity of knowledge management in large companies some colombian}

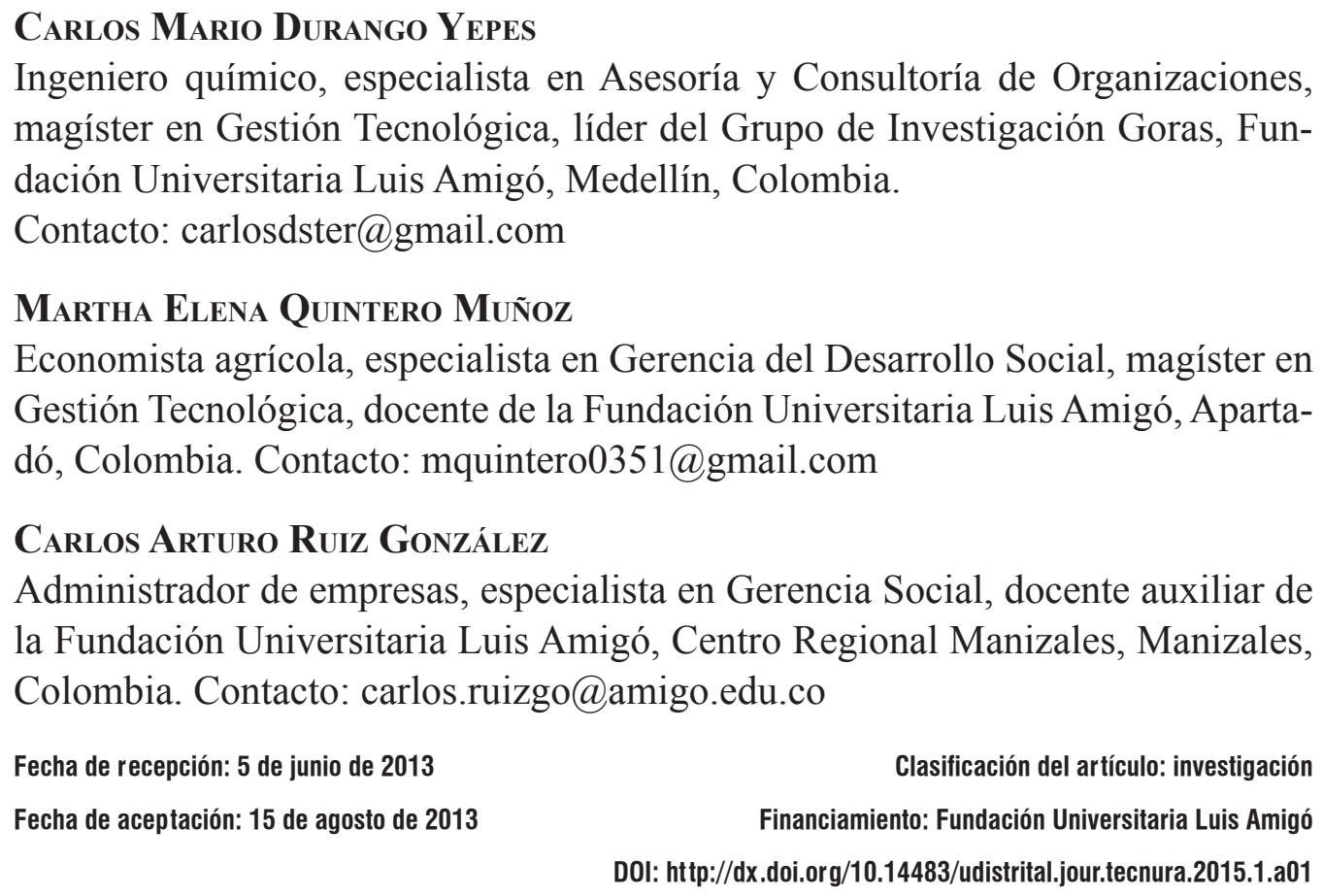

Fecha de recepción: 5 de junio de 2013

Fecha de aceptación: 15 de agosto de 2013

Clasificación del artículo: investigación

Financiamiento: Fundación Universitaria Luis Amigó

DOI: http://dx.doi.org/10.14483/udistrital.jour.tecnura.2015.1.a01

Palabras clave: análisis clúster, epistemologías funcionalista e interpretativa, madurez de la gestión del conocimiento.

Keywords: cluster analysis, functionalist and interpretive epistemologies, maturity of knowledge management.

\section{RESUMEN}

Este artículo propone una metodología para la evaluación de la madurez en la gestión del conocimiento (GC) en trece organizaciones colombianas, desde dos perspectivas epistemológicas: funcionalista e interpretativa, usando de manera exploratoria el análisis clúster para la clasificación de madurez a través de cuatro dimensiones o áreas clave de la gestión del conocimiento (GC), y de todas y cada una de las empresas. Esto permite la medición del nivel de madurez de manera global por empresa y de forma independiente para cada una de las áreas clave, así como comparar las 
empresas según los cinco niveles de la escala de madurez: inicial, conciencia, definido, gestionado y optimizado.

\section{ABSTRACT}

This paper proposes a methodology to assess maturity in Knowledge Management (KM) in thirteen Colombian organizations from two epistemologi- cal perspectives: functionalist and interpretive; using Cluster Analysis in an exploratory manner to classify maturity through four dimensions or key areas of Knowledge Management (KM), in each one of the companies. Allowing the measurement of the maturity level for each company in a global way and independently for each of the key areas. Also, to compare companies according to the five levels in the maturity scale: initial, consciousness, defined, managed and optimized.

\section{INTRODUCCIÓN}

La mayoría de los modelos de madurez para la gestión del conocimiento actualmente en práctica, identifican áreas de proceso clave (o KPA en su sigla en inglés: key process areas) relacionadas con personas, procesos y tecnología. Estas KPA sugieren que la gestión del conocimiento necesita considerar aspectos organizacionales, humanos (por ejemplo, sicológicos y sociológicos) y tecnológicos con el objeto de apoyar de manera exitosa los procesos operativos de dicha gestión (Quintas, Lefrere y Jones, 1997). El área de proceso clave personas incluye aspectos relacionados con la cultura, estrategias y políticas de la organización; el área clave procesos se refiere a aspectos concernientes a los procesos de gestión de conocimiento (GC), tales como creación, recopilación, organización, transferencia y utilización del conocimiento (Durango, 2010); y el área de proceso clave tecnología, se relaciona con la infraestructura tecnológica de soporte a los procesos de GC, aplicaciones o servicios de GC y la actitud hacia las TI para la GC.

El modelo general de madurez de GC, o General Knowledge Management Maturity Model (GKMM en su sigla en inglés) (Pee, Teah y Kankanhalli, 2006), es un modelo descriptivo que describe los atributos esenciales que carac- terizan una organización en un nivel particular de la madurez de la gestión del conocimiento. También es un modelo normativo en el que las prácticas principales caracterizan los tipos ideales de ambiente que deberían esperarse en una organización que esté implementando gestión del conocimiento. En una revisión sobre los fundamentos conceptuales y aspectos de investigación sobre sistemas de GC (Alavi y Leidner, 2001), los autores definen a las organizaciones como sistemas de conocimiento que tienen cuatro procesos de conocimiento: creación (referida como construcción), almacenamiento/recuperación, transferencia, y aplicación (Holsapple y Joshi, 2002; Pentland, 1995). Otros autores identificaron las principales actividades de manipulación del conocimiento: adquisición, selección, interiorización y utilización del conocimiento (Holzner y Marx, 1979) y examinaron los esfuerzos de GC basados en tecnologías de información (TI) en términos de tres procesos: crear, intercambiar y usar el conocimiento (Sabherwal y Sabherwal, 2005).

Para las organizaciones que han implementado alguna forma de GC, el Modelo General de Madurez de GC puede apoyar el desarrollo de la GC mediante el análisis sistemático de su nivel actual de madurez de la GC. El instrumento de evaluación proporcionado por este modelo pue- 
de servir como una herramienta de diagnóstico, ubicando los aspectos que requieren mejora. Esto permite determinar las actividades esenciales y sus prioridades e indica cómo avanzar al siguiente nivel de madurez de GC. Además, puede servir de base para la comparación de las unidades dentro de una organización o entre organizaciones.

Debido al interés investigativo por conocer los avances y los retrocesos en los procesos de implementación de las prácticas de GC en grandes empresas de dos ciudades colombianas intermedias, Manizales y Apartadó, este artículo intenta responder a la pregunta ¿cómo medir el nivel de madurez de la gestión del conocimiento en trece grandes empresas colombianas desde la perspectiva funcionalista e interpretativa?

El texto está organizado de la siguiente manera: primero se desarrolla el concepto de madurez de la GC en el marco del modelo general de madurez; en segundo lugar se considera la madurez de la GC desde la perspectiva interpretativa; en tercer lugar se presenta la metodología; en cuarto lugar se muestran los resultados de medición de la madurez de GC en tres áreas funcionalistas y en el área interpretativa.

\section{MODELO GENERAL DE MADUREZ PARA LA GESTIÓN DE CONOCIMIENTO (GC)}

El primer modelo de madurez, denominado $\mathrm{Ca}$ pability Maturity Model (CMM), fue pensado y aplicado a los procesos de desarrollo de software; este describe e indica por cada uno de sus cinco niveles: inicial, repetible, definido, gerenciado y optimizado (Paulk, Curtis, Chrissis y Weber, 1993), cuáles son las características que marcan la evolución de estos procesos, así como las áreas clave en las que debe implementarse un conjunto de prácticas o focalizarse los cambios en aras de consolidar los mejoramientos. Con ello se definió la estructura básica de los modelos de madurez, a saber: niveles, descripción o características de estos y áreas claves.

La literatura de gestión de conocimiento revela que al igual que el CMM, la mayoría de modelos de madurez de gestión de conocimiento (basados o no en CMM) identifican cinco niveles de madurez. El Modelo General de Madurez de GC propuesto adaptó cinco niveles de madurez de CMM llamados inicial, conciencia, definido, gestionado y de optimización. El nivel 2 fue renombrado por Pee et ál., de "Repetible" a "Conciencia", considerando que "Repetible" es menos intuitivo en el contexto de la gestión de conocimiento y que dicho nivel está principalmente caracterizado por una conciencia de gestionar el conocimiento.

El modelo general de madurez de GC (o General Knowledge Management Maturity Model) es un modelo descriptivo, en el cual se describen los atributos esenciales que caracterizan una organización en un nivel particular de la madurez de la GC. También es un modelo normativo en el que las prácticas principales caracterizan los tipos ideales de ambiente que deberían esperarse en una organización que esté implementando GC.

De manera similar a la mayoría de modelos de madurez de GC basados o no en CMM, el Modelo General de Madurez de GC sigue una estructura por etapas y tiene tres componentes principales, llamados niveles de madurez, áreas de proceso clave o KPA y características comunes. Cada nivel de madurez está compuesto por diferentes KPA, y cada KPA está descrita por un conjunto de características comunes. Estas características especifican las principales prácticas que, cuando son direccionadas colectivamente, ayudan a lograr los objetivos de una KPA. Esta estructura se presenta en la tabla 1. 
Tabla 1. Modelo de madurez de la gestión del conocimiento de corte funcionalista

\begin{tabular}{|c|c|c|c|c|c|}
\hline & \multirow{2}{*}{$\begin{array}{l}\text { Nivel de } \\
\text { madurez }\end{array}$} & \multirow{2}{*}{ Descripción general } & \multicolumn{3}{|c|}{ Áreas clave } \\
\hline & & & $\begin{array}{c}\text { Personas / organiza- } \\
\text { ción }\end{array}$ & Procesos & Tecnología \\
\hline 1 & Inicial & $\begin{array}{l}\text { Poca o ninguna intención } \\
\text { de usar el conocimiento }\end{array}$ & $\begin{array}{l}\text { La organización no es } \\
\text { consciente de la necesi- } \\
\text { dad de GC }\end{array}$ & $\begin{array}{l}\text { No existen procesos } \\
\text { formales para capturar, } \\
\text { compartir y reutilizar el } \\
\text { conocimiento }\end{array}$ & $\begin{array}{l}\text { Sin tecnologías o infra- } \\
\text { estructura de soporte u } \\
\text { apoyo }\end{array}$ \\
\hline 2 & Conciencia & $\begin{array}{l}\text { La organización es cons- } \\
\text { ciente y tiene la intención } \\
\text { de GC, posiblemente no } \\
\text { sepa cómo }\end{array}$ & $\begin{array}{l}\text { Los directivos están } \\
\text { conscientes de la necesi- } \\
\text { dad de GC }\end{array}$ & $\begin{array}{l}\text { Se documentan los } \\
\text { conocimientos indispen- } \\
\text { sables para la realización } \\
\text { de tareas repetitivas }\end{array}$ & $\begin{array}{l}\text { Se han iniciado proyec- } \\
\text { tos piloto de GC (no ne- } \\
\text { cesariamente por iniciati- } \\
\text { va de los directivos) }\end{array}$ \\
\hline 3 & Definido & $\begin{array}{l}\text { La organización ha pues- } \\
\text { to en marcha una infra- } \\
\text { estructura básica que } \\
\text { soporta la GC }\end{array}$ & \begin{tabular}{|l} 
- Se proporciona forma- \\
ción básica sobre GC \\
(por ejemplo: cursos \\
de sensibilización) \\
- Se pone en marcha \\
una estrategia básica \\
de GC \\
- Se han definido roles \\
individuales de GC \\
- Se han activados los \\
sistemas de incentivos
\end{tabular} & $\begin{array}{l}\text { - Se han formalizado } \\
\text { los procesos para la } \\
\text { gestión de contenidos } \\
\text { e información. } \\
\text { - Sistemas de medición } \\
\text { pueden ser usados } \\
\text { para medir el incre- } \\
\text { mento de la producti- } \\
\text { vidad por causa de GC }\end{array}$ & $\begin{array}{l}\text { - Se tiene instalada una } \\
\text { infraestructura básica } \\
\text { de GC (páginas ama- } \\
\text { rillas) } \\
\text { - Se han puesto en mar- } \\
\text { cha algunos proyectos } \\
\text { de GC en algunos } \\
\text { niveles de la pirámide } \\
\text { organizacional }\end{array}$ \\
\hline 4 & $\begin{array}{l}\text { Gestionado } \\
\text { / establecido }\end{array}$ & $\begin{array}{l}\text { Las iniciativas de GC es- } \\
\text { tán planamente estable- } \\
\text { cidas en la organización }\end{array}$ & $\begin{array}{l}\text { - Una estrategia común } \\
\text { y que apunta a la nor- } \\
\text { malización de la GC } \\
\text { - La GC es incorporada } \\
\text { dentro de la estrategia } \\
\text { general de la organi- } \\
\text { zación } \\
\text { - Formación avanzada } \\
\text { en GC } \\
\text { - Estándares organiza- } \\
\text { cionales }\end{array}$ & $\begin{array}{l}\text { Medición cuantitativa de } \\
\text { los procesos de GC (es } \\
\text { decir, el uso de métricas) }\end{array}$ & $\begin{array}{l}\text { - En toda la empresa los } \\
\text { sistemas de GC están } \\
\text { funcionando plena- } \\
\text { mente } \\
\text { - El uso de los sistemas } \\
\text { de GC está en un nivel } \\
\text { razonable } \\
\text { - Perfecta integración } \\
\text { de la tecnología con la } \\
\text { arquitectura de con- } \\
\text { tenidos }\end{array}$ \\
\hline 5 & $\begin{array}{l}\text { Optimizar/ } \\
\text { compartir }\end{array}$ & $\begin{array}{l}\text { La GC está plenamente } \\
\text { integrada a la organiza- } \\
\text { ción y sometida a pro- } \\
\text { cesos de mejoramiento } \\
\text { continuo }\end{array}$ & $\begin{array}{l}\text { La cultura de compartir } \\
\text { esta institucionalizada }\end{array}$ & $\begin{array}{l}\text { - Los procesos de GC } \\
\text { son revisados perma- } \\
\text { nentemente y mejo- } \\
\text { rados } \\
\text { - Los actuales procesos } \\
\text { de GC pueden ser } \\
\text { fácilmente adaptados } \\
\text { para satisfacer las } \\
\text { nuevas necesidades } \\
\text { de negocio } \\
\text { - Los procedimientos de } \\
\text { GC forman parte inte- } \\
\text { gral de la organización }\end{array}$ & $\begin{array}{l}\text { La infraestructura actual } \\
\text { de GC es mejorada con- } \\
\text { tinuamente }\end{array}$ \\
\hline
\end{tabular}

Fuente: Pee, Teah y Kankanhalli (2006). 
Con base en el concepto de organización como sistema de Trist (1981) se pudo llegar a la conclusión de que el subsistema técnico engloba dos de las áreas clave del modelo de madurez, los procesos y la tecnología, y que el subsistema social daría cuenta de la tercera: personas y organización. Sin embargo, de acuerdo con Kast y Rosenzweig (1988), al subsistema técnico y social se le debe agregar el administrativo, que planifica, coordina y controla los flujos de conocimiento en función de las actividades de la organización y su entorno, con el fin de crear unas competencias esenciales (Davenport, 1998).

En suma, el área clave personas y organización comprende la confianza, las habilidades T-shapped, los sistemas de incentivos, la estructura organizacional y la estrategia de GC, donde la confianza se define como la fe en las intenciones y comportamientos de otros y la organización; las habilidades T-Shapped como el grado de comprensión de las tareas propias y de los demás; el sistema de incentivos como las políticas y programas de recompensas económicas o simbólicas que motivan a los empleados a crear y compartir el conocimiento; la estructura organizacional como el grado de implantación y apego de reglas, procedimientos, políticas y estándares; y la estrategia como el foco de la GC hacia las tecnologías o las personas.

Con relación al área procesos de gestión del conocimiento, este trabajo adapta los siguientes conceptos, con base en las propuestas de los autores más representativos (Ruggles, 1997, 1998; Alavi y Leidner, 2001; Holsapple y Joshi, 2002; Sabherwal y Sabherwal, 2005; Lee y Lee, 2007; Zhao, 2010); son ellos:

- La creación de conocimiento es el desarrollo de nuevos conocimientos y procedimientos a partir de patrones, relaciones y significados en datos, información y conocimiento previo.

- La recopilación de conocimiento es la adquisición y el registro de los datos, información, conocimiento en un medio del que se pueden recuperar posteriormente.

- Compartir conocimiento es intercambiar las mejores prácticas entre unidades de una misma organización o entre organizaciones, es dar acceso a las personas que deberían tener acceso a datos, información o conocimiento.

- El uso del conocimiento es llevar datos, información, conocimiento a la tarea que crea valor para una organización.

Finalmente, en el área de tecnologías para la GC, a medida que las organizaciones trabajan para desarrollar y mejorar su sistema de GC, este también cambia de un sistema cerrado, tal como un trabajo en grupo (groupware), o sistema de flujo de trabajo, a un sistema de intercambio de conocimiento en toda la empresa, con más tecnologías inteligentes $\mathrm{y}$, finalmente, a un sistema de intercambio global (Lee y Kim, 2001).

Para entender las variables propias de esta área, se entenderá por servicios de $G C$ a las aplicaciones que usa la empresa, tales como: procesamiento de textos, hojas de cálculo, software de presentación y correo electrónico; páginas amarillas; intranet; portal corporativo; bases de datos internas; datawarehouse; centros de datos (datamarts); groupware; workflow; lecciones aprendidas; inteligencia competitiva; simuladores; minería de datos; sistemas expertos; inteligencia artificial e inteligencia de negocios. Se entenderá por infraestructura tecnológica, la inversión en tecnologías de la información y la comunicación (TIC) que la organización aplica o tiene planificada para soportar las actividades de GC, tales como: instrumentos para la captura; creación y uso compartido de las mejores prácticas; creación de directorios de conocimiento corporativo; localizadores de expertos; páginas amarillas; sistemas de gestión de habilidades; creación de comunidades o redes de conocimiento. Finalmente, se define la actitud hacia las TIC como la disposición que tienen las personas de la organización hacia un 


\section{investigación}

uso escéptico, conservador, vanguardista o innovador de las tecnologías que apoyan las iniciativas de GC.

\section{MODELOS DE MADUREZ DE GESTIÓN DE CONOCIMIENTO (GC) INTERPRETATIVOS}

Los modelos de madurez que se proponen desde esta perspectiva son de naturaleza evolutiva, es decir, comprenden una serie de etapas en las que el nivel de complejidad se incrementa de una a otra, en busca de la perfección. Se deben considerar como modelos de refuerzo que tienen condiciones operativas para maximizar satisfactoriamente los cambios en las organizaciones. Los modelos de madurez, en particular los de naturaleza evolutiva, se caracterizan por la presencia de escenarios óptimos múltiples, que se refieren normalmente a las etapas de desarrollo en el modelo de madurez.

Una propuesta de este tipo de modelos (Desouza, 2006) está conformada por cinco niveles o etapas para evaluar los esfuerzos en GC de la organización, cuya madurez puede describirse en una escala progresiva: predispuesta, reactiva, apreciativa, organizada y optimizada. Los dos componentes de administración del conocimiento que se asignan a la escala de madurez para este trabajo son: significados y acción, donde la gestión de los significados es la manera como se generan y se comparten los significados para el logro de las actividades de la organización, en tanto que la gestión de las actividades se refiere al uso de fuentes de información y de conocimiento que permitan la evaluación de las actividades organizacionales.

En el nivel más básico de este modelo de madurez, el predispuesto, la organización no tendrá una capacidad adecuada para responder o atender a la información proveniente de los entornos internos y externos. Algunas entidades en su interior se podrán especializar en escuchar y responder a la información de los ambientes, pero estarán limitadas al nivel local.

En el nivel reactivo, la organización responde a la presión externa para mejorar la administración del conocimiento; por ejemplo, pone en marcha estrategias para atender y responder las señales en el dominio seleccionado. La organización aprende a manejar mejor la información procedente de las fuentes seleccionadas.

En el nivel apreciativo comienza a valorar la necesidad de entablar acuerdos interdisciplinarios y de análisis transversal para conseguir una mejor apreciación para las fuentes de información, los análisis utilizados para procesarla y los actos de significado y de gestión de la acción.

En el nivel organizado presenta una arquitectura organizada alrededor de la administración de los significados y la administración de la acción. La organización será capaz de llevar a cabo todas las actividades: percepción, disposición para actuar, respuesta guiada, mecanismos, respuestas complejas, adaptación y creación, lo que se puede atribuir a la presencia de una estructura holística de GC.

Finalmente, en el nivel optimizado, se observan similitudes entre los conceptos de personalidad y desarrollo de carácter en la gestión del conocimiento. Con el fin de operar este nivel, la organización se beneficiará de la apertura a la mejora continua y se apoyará en las prácticas y habilidades actuales de los diversos componentes de GC.

\section{METODOLOGÍA}

La medición de la madurez de la GC se realizó en trece empresas que cumplen con el criterio de selección: ser gran empresa y haber implementado iniciativas de GC desde hace por lo menos 5 años. La investigación es correlacional transversal multivariable de campo, para lo cual se utilizó un instru- 


\section{investigación}

mento de recolección de la información ya validado en un trabajo previo realizado en siete grandes empresas en la ciudad de Medellín (Durango, 2010). Adicionalmente, se utilizó el análisis clúster para determinar los rangos de los cinco niveles de madurez, con miras a la clasificación de las empresas. Luego se construyó la base de datos en Excel, asignando un valor de uno a cinco a las cinco opciones de respuesta, uno correspondiente al nivel inicial de la escala de madurez, dos para conciencia, tres para definido, cuatro para gestionado y cinco para optimizado. Además, se promediaron las dieciocho respuestas por empresa, pertenecientes a los niveles operativo, administrativo y gerencial, correspondientes a seis áreas funcionales (tabla 2).
Posteriormente, se procedió a relativizar las variables propias de las cuatro áreas, asignando un valor de prioridad a cada una de ellas, el cual se multiplica por su calificación y se divide por la sumatoria de todas las ponderaciones en el interior de cada una de las dimensiones (Lema, 2002). El valor de prioridad asignado fue de 4 para el área procesos de GC, 3 para el área de tecnologías de apoyo a la GC, 2 al de organización y personas, y 1 a los aspectos interpretativos de la GC. Dicha relativización de las variables se realiza con el ánimo de hacerlas semicuantitativas, para que soporten cualquier análisis multivariado, ya que inicialmente provienen de una escala cualitativa tipo Likert (tabla 3).

Tabla 2. Tabulación de respuestas promedio por variable en cada empresa

\begin{tabular}{|c|c|c|c|c|c|c|c|c|c|c|c|c|c|c|}
\hline \multirow[b]{2}{*}{ Empresas } & \multicolumn{5}{|c|}{ Organización y personas } & \multicolumn{4}{|c|}{ Procesos } & \multicolumn{3}{|c|}{ Tecnología } & \multicolumn{2}{|c|}{$\begin{array}{c}\text { Interpreta- } \\
\text { tivo }\end{array}$} \\
\hline & 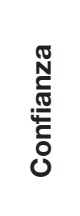 & 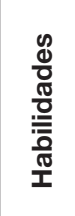 & 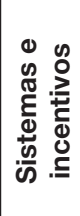 & 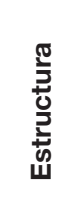 & 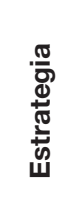 & 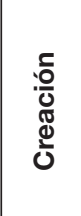 & 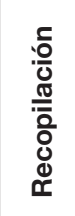 & 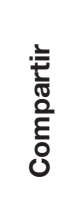 & $\begin{array}{l}: \frac{}{0} \\
\frac{\mathbb{J}}{0} \\
\frac{\overline{0}}{\frac{0}{\alpha}}\end{array}$ & $\begin{array}{l}\text { \& } \\
\frac{0}{0} \\
\text { 응 } \\
\frac{\delta}{0} \\
\frac{0}{0}\end{array}$ & 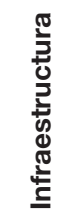 & 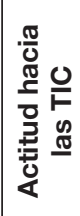 & 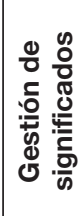 & 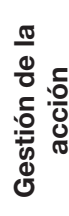 \\
\hline 1. Casa Luker & 2,72 & 3,78 & 2,67 & 2,89 & 3,00 & 0,67 & 2,61 & 2,89 & 2,50 & 1,67 & 2,17 & 2,67 & 1,72 & 1,83 \\
\hline 2. Davivienda & 2,83 & 3,17 & 2,22 & 3,11 & 2,94 & 0,50 & 2,39 & 2,44 & 2,61 & 1,50 & 2,39 & 2,33 & 2,22 & 1,83 \\
\hline $\begin{array}{l}\text { 3. Cooperativa de Caficul- } \\
\text { tores }\end{array}$ & 3,56 & 3,50 & 2,33 & 2,11 & 3,17 & 0,72 & 2,61 & 2,61 & 2,44 & 1,39 & 2,33 & 2,06 & 1,28 & 1,89 \\
\hline 4. Comfamiliares & 3,17 & 3,56 & 1,28 & 1,83 & 1,89 & 0,61 & 2,67 & 2,44 & 2,61 & 1,56 & 2,22 & 2,28 & 1,06 & 1,22 \\
\hline $\begin{array}{l}\text { 5. Suministro de materiales } \\
\text { técnicos }\end{array}$ & 3,39 & 3,78 & 2,33 & 1,61 & 2,00 & 1,17 & 2,17 & 2,61 & 1,50 & 0,89 & 1,28 & 1,94 & 1,78 & 1,67 \\
\hline $\begin{array}{l}\text { 6. Empresa sector textil } \\
\text { manufactura }\end{array}$ & 4,11 & 3,94 & 2,22 & 2,33 & 2,06 & 0,89 & 2,67 & 2,67 & 1,72 & 0,72 & 2,94 & 2,83 & 1,39 & 2,78 \\
\hline 7. Financiera $X$ & 3,44 & 4,00 & 2,28 & 1,72 & 2,50 & 0,83 & 2,28 & 2,61 & 1,83 & 1,56 & 1,67 & 1,72 & 0,61 & 1,83 \\
\hline $\begin{array}{l}\text { 8. Institución de educación } \\
\text { superior }\end{array}$ & 3,22 & 3,56 & 2,61 & 2,33 & 2,17 & 1,00 & 2,22 & 2,28 & 1,50 & 1,33 & 2,72 & 1,44 & 1,39 & 1,67 \\
\hline 9. Telecomunicaciones & 3,72 & 3,89 & 2,94 & 2,94 & 2,83 & 0,89 & 2,17 & 2,17 & 3,00 & 1,06 & 2,22 & 1,94 & 1,78 & 2,39 \\
\hline 10. Financiera Z & 3,28 & 3,56 & 2,61 & 2,33 & 2,22 & 1,50 & 2,44 & 2,28 & 1,50 & 1,33 & 2,56 & 1,44 & 1,17 & 1,78 \\
\hline $\begin{array}{l}\text { 11. Manufactura } \\
\text { electrodomésticos }\end{array}$ & 2,56 & 3,78 & 2,11 & 2,28 & 1,83 & 1,11 & 2,61 & 2,44 & 2,28 & 1,33 & 2,06 & 2,11 & 1,17 & 1,89 \\
\hline 12. Corrugados Del Darién & 3,61 & 4,00 & 2,50 & 1,67 & 2,33 & 0,28 & 2,94 & 2,89 & 2,67 & 1,39 & 2,06 & 2,67 & 1,50 & 1,67 \\
\hline 13. C.I Banacol & 3,11 & 3,44 & 2,06 & 1,46 & 3,00 & 3,28 & 2,94 & 4,17 & 3,33 & 2,33 & 3,17 & 3,72 & 3,28 & 3,11 \\
\hline
\end{tabular}

Fuente: elaboración propia. 
Tabla 3. Variables semicuantitativas del modelo de madurez de GC

\begin{tabular}{|c|c|c|c|c|c|c|c|c|c|c|c|c|c|c|}
\hline \multirow[b]{2}{*}{ EMPRESAS } & \multicolumn{5}{|c|}{ Organización y personas } & \multicolumn{4}{|c|}{ Procesos } & \multicolumn{3}{|c|}{ Tecnología } & \multicolumn{2}{|c|}{$\begin{array}{l}\text { Interpreta- } \\
\text { tivo }\end{array}$} \\
\hline & 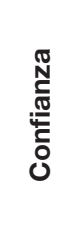 & $\begin{array}{l}0 \\
\frac{0}{0} \\
\frac{\pi}{0} \\
\frac{0}{0} \\
\frac{0}{0} \\
\frac{\pi}{1}\end{array}$ & 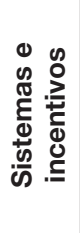 & 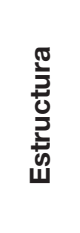 & 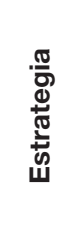 & 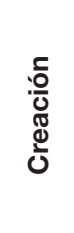 & 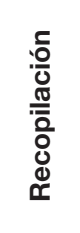 & 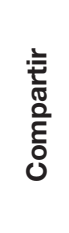 & $\begin{array}{l}: \frac{}{0} \\
\mathbb{8} \\
\frac{0}{0} \\
\frac{0}{\alpha}\end{array}$ & 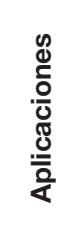 & 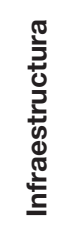 & 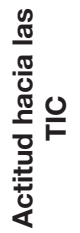 & 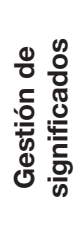 & 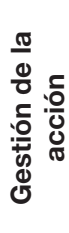 \\
\hline Casa Luker & 0,54 & 0,76 & 0,53 & 0,58 & 0,60 & 0,27 & 1,04 & 1,16 & 1,00 & 0,50 & 0,65 & 0,80 & 0,17 & 0,18 \\
\hline Davivienda & 0,57 & 0,63 & 0,44 & 0,62 & 0,62 & 0,20 & 0,96 & 0,98 & 1,04 & 0,45 & 0,72 & 0,70 & 0,22 & 0,18 \\
\hline $\begin{array}{l}\text { Cooperativa de } \\
\text { Caficultores }\end{array}$ & 0,71 & 0,70 & 0,47 & 0,42 & 0,63 & 0,29 & 1,04 & 1,04 & 0,98 & 0,42 & 0,70 & 0,62 & 0,13 & 0,19 \\
\hline Comfamiliares & 0,63 & 0,71 & 0,26 & 0,37 & 0,38 & 0,24 & 1,07 & 0,98 & 1,04 & 0,47 & 0,67 & 0,68 & 0,11 & 0,12 \\
\hline $\begin{array}{l}\text { Suministro de materiales } \\
\text { técnicos }\end{array}$ & 0,68 & 0,76 & 0,47 & 0,32 & 0,40 & 0,47 & 0,87 & 1,04 & 0,60 & 0,27 & 0,38 & 0,58 & 0,18 & 0,17 \\
\hline $\begin{array}{l}\text { Empresa sector textil } \\
\text { manufactura }\end{array}$ & 0,82 & 0,79 & 0,44 & 0,47 & 0,41 & 0,36 & 1,07 & 1,07 & 0,69 & 0,22 & 0,88 & 0,85 & 0,14 & 0,28 \\
\hline Financiera $\mathrm{x}$ & 0,69 & 0,80 & 0,46 & 0,34 & 0,50 & 0,33 & 0,91 & 1,04 & 0,73 & 0,47 & 0,50 & 0,52 & 0,06 & 0,18 \\
\hline $\begin{array}{l}\text { Institución de educación } \\
\text { superior }\end{array}$ & 0,64 & 0,71 & 0,52 & 0,47 & 0,43 & 0,40 & 0,89 & 0,91 & 0,60 & 0,40 & 0,82 & 0,43 & 0,14 & 0,17 \\
\hline Telecomunicaciones & 0,74 & 0,78 & 0,56 & 0,59 & 0,57 & 0,36 & 1,24 & 0,87 & 1,20 & 0,32 & 0,67 & 0,58 & 0,18 & 0,24 \\
\hline Financiera Z & 0,66 & 0,71 & 0,52 & 0,47 & 0,44 & 0,47 & 0,98 & 0,91 & 0,60 & 0,40 & 0,77 & 0,43 & 0,14 & 0,18 \\
\hline $\begin{array}{l}\text { Manufactura } \\
\text { electrodomésticos }\end{array}$ & 0,51 & 0,76 & 0,46 & 0,46 & 0,37 & 0,44 & 1,04 & 0,98 & 0,91 & 0,40 & 0,62 & 0,63 & 0,12 & 0,19 \\
\hline Corrugados del Darién & 0,72 & 0,80 & 0,50 & 0,33 & 0,47 & 0,11 & 1,18 & 1,16 & 1,07 & 4,17 & 6,17 & 8,00 & 1,50 & 1,67 \\
\hline C.I Banacol & 0,62 & 0,69 & 0,41 & 0,29 & 0,60 & 0,66 & 0,59 & 0,83 & 2,80 & 0,47 & 0,63 & 3,60 & 0,66 & 4,20 \\
\hline
\end{tabular}

Fuente: elaboración propia.

Con las variables relativizadas se construye, por cada una de las empresas y en función de cada una de las cuatro dimensiones, el índice de valor de importancia relativizado (IVIR); un indicador adimensional que muestra el grado de importancia estadística que tiene un dato en un grupo o contexto, según la totalidad de las características de las variables estudiadas (Lema, 2002).
Para calcular el IVIR se suman los valores de las variables pertenecientes a cada una de las dimensiones por cada una de las empresas, y de este modo se construye el índice de valor de importancia (IVI). Luego se le asigna un peso a cada una de las dimensiones, los cuales se multiplican por su correspondiente IVI, se suman los resultados y se dividen entre la sumatoria de las ponderaciones (tabla 4). 


\section{investigación}

Tabla 4. IVI e IVIR de cada una de las empresas

\begin{tabular}{|c|c|c|c|c|c|}
\hline \multirow[b]{2}{*}{ Empresas } & \multicolumn{4}{|c|}{ IVI } & \multirow[t]{2}{*}{ IVIR } \\
\hline & $\begin{array}{l}\text { Organización y } \\
\text { Personas }\end{array}$ & Procesos & Tecnología & Interpretativo & \\
\hline 1. Casa Luker & 3,01 & 3,47 & 1,95 & 0,36 & 2,61 \\
\hline 2. Davivienda & 2,86 & 3,18 & 1,87 & 0,41 & 2,44 \\
\hline 3. Coop. Caficultores Manizales & 2,93 & 3,36 & 1,73 & 0,32 & 2,48 \\
\hline 4. Comfamiliares & 2,34 & 3,33 & 1,82 & 0,23 & 2,37 \\
\hline $\begin{array}{l}\text { 5. Suministro materiales téc- } \\
\text { nicos }\end{array}$ & 2,62 & 2,98 & 1,23 & 0,34 & 2,12 \\
\hline $\begin{array}{l}\text { 6. Empresa sector textil } \\
\text { manufactura }\end{array}$ & 2,93 & 3,18 & 1,95 & 0,42 & 2,48 \\
\hline 7. Financiera $X$ & 2,79 & 3,02 & 1,48 & 0,24 & 2,24 \\
\hline $\begin{array}{l}\text { 8. Institución de educación } \\
\text { superior }\end{array}$ & 2,78 & 2,80 & 1,65 & 0,31 & 2,20 \\
\hline 9. Telecomunicaciones & 3,23 & 3,67 & 1,57 & 0,42 & 2,63 \\
\hline 10. Financiera $Z$ & 2,80 & 2,96 & 1,60 & 0,32 & 2,25 \\
\hline 11. Manufactura & 2,51 & 3,38 & 1,65 & 0,31 & 2,38 \\
\hline 12. Corrugados del Darién & 2,82 & 3,51 & 18,33 & 3,17 & 7,79 \\
\hline 13. C.I Banacol & 2,62 & 4,88 & 4,70 & 4,86 & 4,37 \\
\hline
\end{tabular}

Fuente: elaboración propia.

Este procedimiento se deja en este punto, para dar inicio al análisis clúster que busca clasificar las observaciones de acuerdo con su grado de homogeneidad e identificar los rangos de los cinco niveles de la escala de madurez. Para ello se aplicó la técnica no jerárquica $K$-means, que resulta pertinente cuando se conoce de antemano el número de grupos o conglomerados. En este caso se usó la versión 20 del SPSS, agrupando las observaciones en cuatro conglomerados, porque se asume que ninguna de las empresas se encuentra en un nivel inicial, dado que el estudio se realizó con aquellas que han implementado iniciativas de GC por lo menos con 5 años de antigüedad.

La técnica $K$-means se aplica de manera independiente a cada una de las dimensiones y a sus respectivas variables, asumiendo que las empresas no tienen un nivel de madurez homogéneo. En ocasiones, algunas tienen fortalezas en procesos, pero pueden presentar debilidades en tecnologías o en otras áreas, y viceversa.
Consecutivamente, se suman los valores que arroja el análisis clúster de las variables de cada dimensión, para generar el IVI de cada uno de los cuatro conglomerados (ver tabla 5), el cual se utiliza para clasificarlos de menor a mayor en conciencia, definido, gestionado y optimizado.

Tabla 5. IVI de los conglomerados por cada una de las dimensiones del modelo de madurez de GC

\begin{tabular}{|l|c|c|c|c|}
\hline \multirow{2}{*}{ Dimensión } & \multicolumn{4}{|c|}{ Conglomerados } \\
\cline { 2 - 5 } & $\begin{array}{c}\text { Clúster } \\
\mathbf{1}\end{array}$ & $\begin{array}{c}\text { Clúster } \\
\mathbf{2}\end{array}$ & $\begin{array}{c}\text { Clúster } \\
\mathbf{3}\end{array}$ & $\begin{array}{c}\text { Clúster } \\
\mathbf{4}\end{array}$ \\
\hline $\begin{array}{l}\text { Organización } \\
\text { y personas }\end{array}$ & 2,82 & 2,8 & 3,06 & 2,43 \\
\hline Procesos & 3,51 & 3,02 & 3,50 & 3,36 \\
\hline Tecnología & 18,33 & 1,63 & 1,75 & 1,73 \\
\hline Interpretativa & 3,17 & 0,34 & 0,36 & 0,27 \\
\hline
\end{tabular}

Fuente: elaboración propia. 


\section{investigación}

Lo anterior sirve de base para estimar los rangos de los cinco niveles de la escala de madurez (ver tabla 6) y determinar en cuál se encuentran las empresas, al ubicar en alguno de ellos el IVI de las cuatro dimensiones.

Si a la tabla 5 se le ponen los pesos dados en la relativización de las variables se obtiene la tabla 7.

Con base en la tabla 7 se puede calcular el IVIR conglomerado para cada área clave, sumando el producto de los IVI de cada área por su respectivo peso y dividiendo entre la suma de los pesos, o sea entre 10. Así se obtienen los valores de la tabla 8 .

La tabla 9 se obtiene a partir de la tabla 8 , ordenando los cuatro IVIR conglomerados en cinco rangos o niveles de madurez.

Seguidamente, comparando los IVIR obtenidos para cada empresa en la tabla 4 y ubicándolas en los cinco rangos de madurez definidos en la tabla 9, se determina la ubicación de cada empresa en cada nivel de madurez ( tabla 10).

Tabla 6. Rangos de los niveles de madurez por cada una de las dimensiones del modelo de madurez de gestión de conocimiento

\begin{tabular}{|l|c|c|c|c|c|}
\hline \multicolumn{1}{|c|}{ Áreas clave } & Inicial & Consciencia & Definido & Gestionado & Optimizado \\
\hline Organización y Personas & $0 \leq n<2,43$ & $2,43 \geq n<2,8$ & $2,8 \geq n<2,82$ & $2,82 \geq n<3,06$ & $n \geq 3,06$ \\
\hline Procesos & $0 \leq n<3,02$ & $3,02 \geq n<3,36$ & $3,36 \geq n<3,50$ & $3,50 \geq n<3,51$ & $n \geq 3,51$ \\
\hline Tecnología & $0 \leq n<1,63$ & $1,63 \geq n<1,73$ & $1,73 \geq n<1,75$ & $1,75 \geq n<18,33$ & $n \geq 18,33$ \\
\hline Interpretativo & $0 \leq n<0,27$ & $0,27 \geq n<0,34$ & $0,34 \geq n<0,36$ & $0,36 \geq n<3,17$ & $n \geq 3,17$ \\
\hline
\end{tabular}

Fuente: elaboración propia.

Tabla 7. IVI de los conglomerados con sus pesos relativos

\begin{tabular}{|l|c|c|c|c|c|}
\hline \multirow{2}{*}{\multicolumn{1}{c|}{ Áreas clave }} & \multicolumn{5}{c|}{ IVI conglomerados } \\
\cline { 2 - 6 } & Peso & Clúster 1 & Clúster 2 & Clúster 3 & Clúster 4 \\
\hline Organización y Personas & 2 & 2,82 & 2,80 & 3,06 & 2,43 \\
\hline Procesos & 4 & 3,51 & 3,02 & 3,50 & 3,36 \\
\hline Tecnología & 3 & 18,33 & 1,63 & 1,75 & 1,73 \\
\hline Interpretativo & 1 & 3,17 & 0,34 & 0,36 & 0,27 \\
\hline & 10 & & & & \\
\hline
\end{tabular}

Fuente: elaboración propia.

Tabla 8. IVIR conglomerado

\begin{tabular}{|l|c|}
\hline \multicolumn{1}{|c|}{ Áreas clave } & IVIR conglomerado \\
\hline Organización y Personas & 2,22 \\
\hline Procesos & 5,35 \\
\hline Tecnología & 7,03 \\
\hline Interpretativo & 0,41 \\
\hline
\end{tabular}

Fuente: elaboración propia.
Tabla 9. Construcción de los rangos de madurez por áreas clave para cada nivel de madurez

\begin{tabular}{|l|c|}
\hline \multicolumn{1}{|c|}{ Nivel de madurez } & Rango de madurez \\
\hline Inicial & $0 \leq \mathrm{n}<0,41$ \\
\hline Consciencia & $0,41 \geq \mathrm{n}<2,22$ \\
\hline Definido & $2,22 \geq \mathrm{n}<5,35$ \\
\hline Gestionado & $5,35 \geq \mathrm{n}<7,03$ \\
\hline Optimizado & $\mathrm{n} \geq 7,03$ \\
\hline
\end{tabular}

Fuente: elaboración propia. 
Finalmente, la posición de cada empresa en cada área clave (tabla 11) se logra si se ubican los IVI resultantes de la tabla 4 en los rangos de los niveles de madurez por cada área clave obtenidos en la tabla 6 .

\section{ANÁLISIS DE LOS RESULTADOS}

\section{Área clave Organización y Personas}

La única empresa que se encuentra en el nivel inicial en esta área es la número 4: Comfamiliares, lo que de acuerdo con el Modelo General de GC significa que no es aun consciente de la necesidad de GC. Ubicadas en el nivel de madurez consciente están cinco empresas, que representan, el 38,5\% de la población objeto de estudio, correspondiente a dos empresas comercializadoras, una entidad financiera $X$, una institución de educación superior y una empresa de manufactura. Los directivos están conscientes de la necesidad de GC. Por otra parte, está otra institución financiera (denominada Z, por reserva de su nombre), así como Corrugados del Darién - clasificadas en el nivel de madurez definido-, donde se llevan a cabo acciones de sensibilización en $\mathrm{GC}$, se pone en marcha una estrategia básica de $\mathrm{GC}$, se han definido roles individuales de GC o se han activado sistemas de incentivos. Cuatro empresas se ubican en el nivel de madurez gestionado, estas son: Casa Luker, Davivienda, Cooperativa de Caficultores de Manizales y una empresa del sector textil, donde la GC es incorporada dentro de la estrategia general de la organización, es evaluada en los estándares organizacionales y hay una formación avanzada en el tema. La empresa mejor ubicada, en el nivel de madurez optimizado, es de telecomunicaciones, ubicada en Medellín, donde la cultura de compartir el conocimiento esta institucionalizada.

Tabla 10. Ubicación de cada empresa en cada nivel de madurez

\begin{tabular}{|l|l|}
\hline \multicolumn{1}{|c|}{ Nivel de madurez } & Empresa \\
\hline Inicial & \\
\hline Conciencia & 5,8 \\
\hline Definido & $1,2,3,4,6,7,9,10,11$ \\
\hline Gestionado & 13 \\
\hline Optimizado & 12 \\
\hline
\end{tabular}

Fuente: elaboración propia.

Tabla 11. Ubicación de cada empresa según área clave

\begin{tabular}{|l|l|l|l|l|l|}
\hline \multirow{2}{*}{\multicolumn{1}{c|}{ Áreas clave }} & \multicolumn{5}{c|}{ Nivel de madurez } \\
\cline { 2 - 6 } & \multicolumn{1}{|c|}{ Inicial } & \multicolumn{1}{c|}{ Conciencia } & \multicolumn{1}{c|}{ Definido } & \multicolumn{1}{c|}{ Gestionado } & \multicolumn{1}{c|}{ Optimizado } \\
\hline Organización y Personas & 4 & $5,7,8,11,13$ & 10,12 & $1,2,3,6$ & 9 \\
\hline Procesos & $5,8,10$ & $2,4,6,7$ & $1,3,11$, & & $9,12,13$ \\
\hline Tecnología & $5,7,9,10$ & 8,11 & 3 & $1,2,4,6,13$ & 12 \\
\hline Interpretativo & 4,7 & $3,8,10,11$ & 5 & $1,2,6,9$ & 12,13 \\
\hline
\end{tabular}

Fuente: elaboración propia. 


\section{Área de Procesos de gestión de conocimiento y tecnología}

En el caso de las empresas número 5 (distribución comercial), número 8 (institución de educación superior) y número 10 (Financiera Z), los hallazgos hechos de acuerdo con la información suministrada permiten categorizarlas en un estado de madurez inicial con relación a los procesos de creación, recopilación, organización, transferencia y aplicación del conocimiento, de la siguiente manera:

- Las personas desarrollan nuevos conocimientos sin que la organización defina en esta materia reglas, procedimientos, políticas y estándares.

- No existen procesos formales para identificar, adquirir y registrar los datos, la información y el conocimiento.

- No hay estructura formal para clasificar el conocimiento.

- El intercambio, la exteriorización, la interiorización de conocimiento y la formación son actividades individuales.

El conocimiento es utilizado solo por el individuo para llevar a cabo una actividad basada en conocimiento. El conocimiento que se produce, por lo general, no está disponible para otras personas.

Las empresas número 5 (distribución comercial) y número 10 (Financiera Z) también se ubican en la categoría inicial con relación a los aspectos tecnológicos que apoyan los procesos de GC, compartiendo esta ubicación con las empresas número 7 (Financiera $\mathrm{X}$ ) y número 9 (telecomunicaciones). Las características de una organización en dicho estado muestran las siguientes condiciones en su infraestructura tecnológica, en sus prácticas de GC y en su actitud hacia las TIC:
- La organización no cuenta con TIC para llevar a cabo actividades de GC y la existente no se usa para este propósito.

- Las aplicaciones de GC usan procesamiento de textos, hojas de cálculo, software de presentación y correo electrónico.

\section{LAS PERSONAS SON ESCÉPTICAS, SIN CONOCIMIENTO BÁSICO DE LAS TI}

En la categoría conciencia o repetible del modelo de madurez para el área de procesos de GC, se encuentran las empresas número 2 (Davivienda), número 4 (Comfamiliares), número 6 (empresa del sector textil manufactura) y número 7 (Financiera X). Lo cual puede significar lo siguiente:

- Las personas desarrollan nuevos conocimientos y documentan lo que es indispensable para la realización de sus tareas.

- Se documentan los conocimientos indispensables para la realización de tareas repetitivas.

- Se investiga qué procesos se tienen y se requieren para clasificar el conocimiento y la información.

- La organización le da importancia a los diversos mecanismos para compartir y difundir conocimientos, pero aún no ha elaborado un plan de transferencia.

En esta misma categoría (conciencia) del modelo de madurez, pero para el área de tecnología, se encuentra las empresas número 8 (institución de educación superior) y número 11 (manufactura). Lo cual puede interpretarse de la siguiente manera:

- En algunas áreas de la organización se usan las TIC existentes para iniciativas o proyectos piloto de GC.

- Las páginas amarillas e intranets son las aplicaciones propias de GC. 
- En su actitud hacia las TIC son conservadores, con conocimiento básico de la TI, o en periodo de aprendizaje o entrenamiento inicial.

Las empresas número 1 (Casa Luker), número 3 (Cooperativa de Caficultores de Manizales) y número 11 (Manufactura) comparten el nivel definido en el área de procesos. Para el área procesos ubicarse en este nivel puede interpretarse de la siguiente manera:

- Las personas desarrollan nuevos conocimientos mediante métodos y estrategias definidas por la organización.

- Se han formalizado los procesos para la gestión de contenidos y de la información.

- Se selecciona el conocimiento que se posee en los procesos que se van a trabajar según su importancia y aplicación.

- La organización implementa un plan para la transferencia de conocimientos.

- El conocimiento es usado por el equipo de tomadores de decisión mediante el uso del conocimiento individual.

Adicionalmente, la empresa número 3 (Cooperativa de Caficultores de Manizales) se encuentra en este mismo nivel en la dimensión tecnología. Para el área tecnología, ubicarse en el nivel definido puede interpretarse de la siguiente manera:

- La organización tiene una infraestructura básica de GC que puede ser accedida a través de la intranet o portal corporativo.

- Bases de datos internas, almacenes normalizados (data warehouse, centros de datos (data marts), group ware, work flow.

- Adoptadores tempranos, con un nivel medio de conocimiento o dominio de TI en las actividades de GC
La única empresa que se encuentra en el nivel gestionado en el área de tecnología es C. I. Banacol, lo cual se puede interpretar de la siguiente manera:

- Medición cuantitativa de los procesos de GC (es decir, el uso de métricas).

- En toda la empresa las aplicaciones de GC están perfectamente integrados a los procesos de negocio de la empresa.

- Transferencia interna de conocimiento, venta de conocimiento, lecciones aprendidas, inteligencia competitiva.

- Promotores, con un nivel medio y fuerte de conocimiento o dominio de TI en las actividades de GC en toda la organización.

Las únicas empresas en el nivel de madurez optimizado para el área de procesos de GC es la empresa número 9 (telecomunicaciones), la número 12 (Corrugados del Darién) y la número13 (C. I. Banacol), lo cual puede ser interpretado de la siguiente manera:

- Las personas desarrollan nuevos conocimientos respondiendo a las demandas del entorno, y la organización mejora permanentemente los métodos y estrategias para el desarrollo de nuevos conocimientos.

- Retroalimentación y planes de mejoramiento a los procesos de identificación, adquisición y selección del conocimiento.

- Se reestructura la clasificación de los activos de conocimiento que se ha optimizado.

- El proceso de transferencia de conocimiento es constantemente revisado y mejorado, y puede adaptarse fácilmente a las nuevas necesidades del negocio.

- El proceso de aplicación del conocimiento a sus actividades misionales es constantemente revisado y mejorado 
Adicionalmente, la empresa número 12 (Corrugados del Darién) se ubica en el nivel optimizado en el área tecnología. Esta ubicación podría ser interpretada de la siguiente manera:

- La infraestructura tecnológica para las aplicaciones de GC integrada a los procesos de negocio se mejora continuamente.

- Las aplicaciones de GC usan sistemas expertos, inteligencia artificial o la inteligencia de negocios.

- La actitud hacia las TIC los caracteriza como innovadores, con conocimientos avanzados de la TI y sus aplicaciones actuales y potenciales.

\section{Área interpretativa de la gestión de conocimiento}

Desde el enfoque interpretativo se asume que el conocimiento es una construcción de la experiencia humana y de las prácticas sociales de conocimiento, por lo cual, en esta se ahonda en aspectos subjetivos e intersubjetivos de las personas o sujetos que hacen parte de las organizaciones.

Las empresas número 4 (Comfamiliares) y número 7 (Financiera X) se encuentran en el nivel inicial del enfoque interpretativo, lo cual puede evidenciar lo siguiente:

- Los individuos crean significados pero estos son incompletos y rara vez son compartidos de forma efectiva.

- Habrá un vacío en la generación y la aplicación de conocimiento, cuyo resultado serán acciones poco calibradas.

- Las acciones se derivan de los pálpitos individuales, y no hay retroalimentación ni la posibilidad de monitorear su efectividad.

Las empresas número 3 (Cooperativa de Caficultores de Manizales), número 8 (institución de educación superior), número 10 (Financiera Z) y número 11 (manufactura) se encuentran en el nivel de conciencia del enfoque interpretativo, lo cual puede evidenciar que:

- Los significados son compartidos dentro de los centros y emerge un lenguaje común.

- Los significados son también intercambiados, comparados, debatidos (retroalimentación), buscando mejorar las métricas que anteceden y se requieren para las acciones.

- La gestión de la retroalimentación de las acciones será restringida en estos centros.

- Las acciones calibradas dentro de los centros se basan en los significados, lo cual aumenta sus niveles de eficiencia y flexibilidad.

La empresa número 5 (Suministro de Materiales Técnicos) se encuentra en el nivel definido, lo cual puede tener la siguiente significancia:

- Los significados son compartidos en las funciones de la organización.

- Este proceso demanda traductores para llegar a acuerdos en la sintaxis y la terminología.

- En los centros se comienza a incorporar experiencias externas en las prácticas de trabajo. Los centros en alianza ejecutan acciones más comprensivas y obtienen retroalimentación de sus acciones más allá del contexto (desde afuera de los centros).

- Ello repercute en la eficiencia y genera cambios en aras de mayor efectividad

Las empresas número 1 (Casa Luker), número 2 (Davivienda) y número 6 (empresa sector textil) se encuentran en el nivel gestionado del enfoque interpretativo, lo cual tiene la siguiente significancia en la GC para estas organizaciones:

- Los significados basados en lo analítico son profundos y se comparten con los miembros de la organización. 
- La organización gestiona la diversidad en los lenguajes organizacionales, al tiempo que promueve diálogos basados en significados.

- La organización diseña acciones únicas basadas en nuevos significados.

- Se habilita un repositorio de nuevas acciones, facilitando la reutilización del conocimiento existente.

- La retroalimentación es efectiva y ayuda a la organización a la evaluación de sus acciones.

Las empresas número 12 (Corrugados del Darién) y número 13 (C. I. Banacol), se encuentran en el nivel optimizado del enfoque interpretativo, lo cual tiene la siguiente significancia para ambas organizaciones:

- Se habilitan mecanismos para revisar constantemente los significados generados.

- Existe el compromiso de entrenar a los individuos para que conserven sus habilidades, así como el compromiso de mantener mecanismos compatibles y eficientes.

- Las acciones son revisadas periódicamente; las operaciones de la organización se mantienen al día.

- Las métricas para la evaluación de las acciones son mejoradas continuamente, buscando que sea en tiempo real.

- La organización cuenta con repositorio de acciones que es preciso seguir, el cual es actualizado regularmente.

\section{CONCLUSIONES}

- El modelo de madurez de la GC es sistemático y apropiado para la evaluación de las áreas clave, lo cual permite mostrar los resultados de la medición del nivel de madurez de manera global o de forma independiente para cada una de las áreas clave. Esto asimismo hace posible precisar que la empresa Corrugados del Darién, seguida de la empresa de telecomunicaciones y de C. I. Banacol son las que están mejor ubicadas en las cuatro áreas de GC consideradas.

- Esta metodología proporciona un algoritmo de cálculo que permite semicuantitativizar variables cualitativas provenientes de una escala Likert, y obtener en cada clúster definido previamente, el índice de valor de importancia (IVI), al igual que el valor global del IVI (o sea el IVIR) para las cuatro dimensiones y para cada empresa objeto del estudio. Este algoritmo es similar al propuesto en el Modelo Navegador del Conocimiento (KNM, en su sigla en inglés).

- Los datos cualitativos analizados y utilizados para la construcción de un modelo exploratorio son significativos, ya que se obtienen de los niveles operativo, administrativo y gerencial, que desde hace cinco o más años han implementado prácticas relacionadas con la GC.

- El instrumento en su conjunto está diseñado para posicionarse como un instrumento de autoevaluación fácilmente entendible, que posteriormente pueda ser puesto en línea para su comparabilidad con empresas que se configuren como de talla mundial.

\section{TRABAJOS FUTUROS}

Luego de diseñado el modelo y aplicado en una versión preliminar, como parte de una segunda fase de la investigación, se está realizando durante el 2013 el mismo trabajo, pero ampliando la muestra a por lo menos 30 empresas, para proponer posteriormente un modelo exploratorio propiamente dicho para grandes empresas colombianas. Posteriormente, se trabajará la relación madurez de GC e innovación en productos académicos universitarios. 


\section{FINANCIAMIENTO}

Este artículo es producto de la investigación $M a$ durez de la gestión del conocimiento en grandes empresas del país, realizada en 2012, la cual ha sido avalada y financiada como proyecto de mediana cuantía por la Funlam.

\section{REFERENCIAS}

Alavi, M. y Leidner, D. E. (2001). Review: Knowledge Management and Knowledge Management Systems: Conceptual Foundations and Research Issues. MIS Quarterly, 25 (1), 107-136.

Bloom, B., Englehart, M., Furst, E, Hill, W. y Krathwohl, D. (1956). Taxonomy of Educational Objectives: The Classification of Educational Goals. En: Handbook I: Cognitive domain. Nueva York, Toronto: Longmans, Green.

Davenport, T. y Grover, V. (2001). Special Issue: Knowledge Management. Journal of Management Information Systems, 31 (7), 7-10.

Davenport, T. y Prusak, L. (1998). Working Knowledge: How Organizations Manage what they Know. Estados Unidos: Harvard Business School Press.

Desouza, K. (2006). Knowledge Management Maturity Model: Theoretical Development and Preliminary Empirical Testing (tesis doctoral). University of Illinois at Chicago.

Durango, C. (2010). Construcción de una herramienta para el diagnóstico de la madurez de los procesos de gestión de conocimiento. Informe avance Funlam, junio de 2010. Documento inédito.

Durango, C. y Arias, J. (2010). Madurez de los procesos y tecnologías de gestión del co- nocimiento en empresas industriales de Antioquia. En Memorias Acacia 2012, Atizapán, México.

Holsapple, C. W. y Joshi, K. D. (2002). Knowledge Manipulation Activities: Results of a Delphi study. Information \& Management, 39 (6), 477-490

Holzner, B. y Marx, J. (1979). Knowledge Application: The Knowledge System in Society. Boston: Allyn \& Bacon.

Hsieh, P. J., Lin, B. y Lin, C. (2009). The Construction and Application of Knowledge Navigator Model (KNM TM): An Evaluation of Knowledge Management Maturity. Expert Systems with Applications: An International Journal, 36 (2), 4087-4100.

Kast, F. y Rosenzweig, J. (1988). Administración en las organizaciones. Enfoque de sistemas $y$ de contingencias. México: McGraw-Hill/ Interamericana de México.

Lee, Y. y Lee, S. (2007). Capabilities, Processes, and Performance of Knowledge Management: A Structural Approach. Human Factors and Ergonomics in Manufacturing, 17 (1) $21-41$.

Lee, J. y Kim, Y. (2001). A Stage Model of Organizational Knowledge Management: A Latent Content Analysis. Expert Systems with Application, 20 (4), 299-311. 
Lema, A. (2002). Elementos de estadística multivariada. Medellín: Silvano.

Nonaka, I. (1991). The Knowledge Creating Company. Harvard Business Review, 69 (6), 96-104.

Paulk, M., Curtis, B., Chrissis, M. y Weber, C. (1993). Capability Maturity Model for Software, Version 1.1. Technical Report CMU/SEI-93-TR-024. Software Engineering Institute, Carnegie Mellon University.

Pee, L., Teah, H. y Kankanhalli, A. (2006). Development of a General Knowledge Management Maturity Model. Ponencia presentada en Korean Knowledge Management Society Conference (pp. 17-18), Seúl, Corea del Sur.

Pentland, B. T. (1995), Grammatical Models of Organizational Processes. Organization Science, 6, 541-556.

Quintas, P., Lefrere P. y Jones, G. (1997). Knowledge Management: A Strategic Agenda. Longe Range Planning, 30, 385-391.

Ramaprasad, A. y Ambrose, P. (1999). The Semiotics of Knowledge Management. Proceedings, WITS, Charlotte, NC.
Ruggles, R. (1997). Knowledge Tools: Using Technology to Manage Knowledge Better. Recuperado de http://www.businessinnovation.ey.com $/ \mathrm{mko} / \mathrm{html} /$ toolsrr.html

Ruggles, R. (1998). The State of the Notion: Knowledge Management in Practice. California Management Review, 40 (3), 80-89.

Saussure. F (1916). Course in General Linguistics. Nueva York: McGraw-Hill Book Company.

Sabherwal, R. y Sabherwal, S. (2005). Knowledge Management Using Information Technology: Determinants of Short-Term Impact on Firm Value. Decision Sciences, $36(4), 531-567$.

Trist, E. (1981). The Evolution of Socio-Technical Systems. Toronto, Ontario: Quality of Working Life Centre.

Zhao, J. (2010). School Knowledge Management Framework and Strategies: The New Perspective on Teacher Profesional Development. Computers in Human Behavior, 26, 168-175. 